\title{
Nación y territorio. Análisis comparado del sentimiento nacionalista y la pertenencia a territorios en Andalucía y Cataluña
}

\author{
Livia García Faroldi \\ Universidad de Málaga. Área de Sociología \\ lgarcia@uma.es
}

Recibido: 15-01-2008

Aceptado: 12-05-2009

\section{Resumen}

El presente trabajo compara el sentimiento nacionalista y el territorio con el que se siente identificado en primer lugar el individuo en dos Comunidades, Andalucía y Cataluña. El estudio se inscribe dentro de una investigación más amplia que analiza las estructuras sociales de ambas Comunidades y que supone, siguiendo la hipótesis de la teoría de la modernización, que en las sociedades modernas las categorías sociales predicen peor los comportamientos del individuo que en sociedades menos individualistas. Se trata de comprobar si las variables independientes (edad, sexo, nivel educativo, posición social, estatus ocupacional, ideología, tamaño del hábitat y postmaterialismo) explican mejor el sentimiento nacionalista y la pertenencia a un territorio en Andalucía que en Cataluña y en 1995 (cuando las sociedades eran menos modernas) que en 2006. Los resultados muestran que las variables independientes explican mejor el comportamiento de los catalanes y que, además, la mayoría han aumentado su capacidad explicativa en la última década, especialmente la ideología y los valores postmaterialistas.

Palabras clave: nacionalismo, identificación geográfica, modernización política.

\section{Abstract. Nation and territory. Comparative analysis of nationalist feeling and geographical belonging in Andalusia and Catalonia}

This article compares nationalist sentiment and the first choice of geographical identification of individuals in Andalusia and Catalonia. This research is part of a wider project which analyses social structures in both communities. Following the modernization theory's hypothesis, this project asserts that social behaviour is worse predicted by social categories in modern societies than in less individualistic ones. We checked if independent variables (age, sex, educational level, social position, social occupation, ideology, size of habitat and index of postmaterialist values) explain better nationalist feeling and geographical belonging better in Andalusia than in Catalonia, and also better in 1995 (when both societies were less modern) than in 2006. Results show that independent variables predict to a greater extent the Catalonians' behaviour and, indeed, most of the variables have gained significance in last decade, especially ideology and postmaterialist values.

Keywords: nationalism, geographical belonging, political modernization. 


\section{Sumario}
1. Marco teórico: la teoría de la modernización y su aplicación
4. Evolución y comparación del sentimiento nacionalista (1995 y 2006) al estudio del sentimiento nacionalista y de pertenencia a territorios
2. Metodología
5. Conclusiones
6. Referencias bibliográficas
3. Comparación del espacio con el que se siente más identificado (2006)

\section{Marco teórico: la teoría de la modernización y su aplicación al estudio del sentimiento nacionalista y de pertenencia a territorios}

El presente trabajo se enmarca en otro más amplio dedicado al estudio del cambio social en Andalucía ${ }^{1}$. Se parte del enfoque propuesto por la teoría de la modernización de Donald Treiman (1970): en una sociedad moderna, en la que el proceso de individuación está más avanzado que en una sociedad menos desarrollada, el comportamiento del actor es más difícil de predecir porque las categorías sociales a las que pertenece apenas explican su comportamiento. Obviamente, la modernización así considerada es un concepto relativo, pues no existe sociedad completamente moderna, por ello se la entiende como un proceso más que un estado. La noción de modernización ha estado impregnada de una fuerte carga ideológica en los años sesenta y setenta y se la ha acusado, especialmente en el uso que se le ha dado en los estudios funcionalistas, de caer en el relativismo, el endogenismo y el etnocentrismo (para un análisis de estas críticas y una definición operativa que tiene en cuenta la acción de los agentes sociales sobre las estructuras, ver Solé, 1976). Con el paso de los años y la constatación de que la evolución social tomaba nuevas vías, este concepto se ha etiquetado de "simple» y se habla hoy de modernización reflexiva (ver, por ejemplo, Beck, 1992, y el análisis que realiza Solé, 1997, sobre los vínculos entre modernización, modernidad y riesgo).

Este trabajo intenta comprobar la hipótesis de la teoría de la modernización "clásica» - que habitualmente centra su atención en aspectos socioeconómicos del desarrollo_-, estudiando dos variables de carácter político, relacionadas con las identidades colectivas y que, a su vez, están asociadas en cierta medida entre sí: por un lado, cuál es el colectivo político con el que se siente más identificado el individuo (la variable «sentimiento nacionalista») y, por otro, cuál es el espacio geográfico con el que se siente más identificado (variable "primer espacio con el que se siente identificado»).

El estudio del nacionalismo ha generado diversas corrientes teóricas contrapuestas sobre su definición, alcance, tipología e incluso consecuencias. Sin

1. El trabajo global está subvencionado por la Fundación Centro de Estudios Andaluces (Ref. SOC1.07/023), entidad dependiente de la Consejería de la Presidencia de la Junta de Andalucía. 
embargo, existe un cierto consenso entre autores consagrados en el tema a la hora de afirmar que entre el nacionalismo y la modernización existe una relación causal, porque el primero es la respuesta a un proceso de desintegración de las comunidades tradicionales en Europa. Hobsbawm (1992) remarca que la nación difiere en tamaño, escala y naturaleza de las comunidades con las que los hombres se han identificado la mayor parte de la historia, y le hace demandas diferentes. En su origen, el estado moderno se enfrentó a dos problemas políticos: uno técnico-administrativo para vincular a cada habitante con el gobierno, y otro para afrontar cómo desarrollar la lealtad ciudadana y la identificación con el estado y el sistema de normas. Los estados requerían una religión cívica, un patriotismo, cuya idea original estaba basada en el estado más que en el nacionalismo, puesto que se relacionaba con el pueblo soberano. Sin embargo, los estados reforzaron el patriotismo estatal con sentimientos y símbolos de una "comunidad imaginada» (en términos de Anderson, 1983, quien la define como una comunidad política imaginada, imaginada además como limitada y soberana), dondequiera y comoquiera que se hubieran originado, y usaron su maquinaria de comunicación, sobre todo la escuela primaria, para extender la imagen y la herencia de la nación e inculcar una ligadura al país y a la bandera, a menudo «inventando tradiciones» o incluso naciones.

La importancia que los autores han otorgado a la intelligentsia en este proceso ha sido diferente: mientras que Hayes (1966), Kedourie (1961) y Deutsch (1981) destacan el papel de las minorías intelectuales para difundir el sentimiento nacionalista, Smith $(1986,1991)$ resalta que, aunque establecer una identidad nacional puede ser un proyecto político al que aspiren las élites políticas, las naciones se construyen sobre un fundamento premoderno, un núcleo étnico (core ethnie): los mitos, las memorias, los valores y los símbolos sirven para crear la cultura nacional. También algunos autores han destacado el papel del componente humano, de la intelligentsia autóctona, en la difusión de innovaciones científicas y tecnológicas que conlleva la modernización, teniéndola en cuenta en la definición que se realiza de ésta (Solé, 1976). En este trabajo, se prestará atención al perfil de los que se declaran nacionalistas en ambas comunidades, para averiguar si ocupan posiciones sociales prestigiosas en su sociedad, es decir, si forman parte de la élite económica, política y cultural.

La segunda variable mencionada, el primer espacio con el que se siente identificado el individuo, muestra una relación importante con el sentimiento nacionalista. Sin embargo, la relación no es perfecta, porque existen muchas más posibles categorías de respuesta en esta variable (un total de siete espacios, más la respuesta «ninguno») que en la del sentimiento nacionalista (cinco categorías que se han recodificado posteriormente en tres). En cuanto a cómo interpreta la teoría de la modernización el espacio geográfico con el que se siente identificado el individuo, resulta aquí pertinente recordar la conocida teoría de la revolución silenciosa de Inglehart $(1977,1990)$. Este autor distingue entre dos orientaciones, materialista y postmaterialista, y afirma que: 1) los materialistas son menos cosmopolitas, tienen una mente más estrecha que los postmaterialistas, y 2) los postmaterialistas están más deseosos de lle- 
nar una cierta necesidad de pertenencia, una necesidad que puede satisfacerse mejor identificándose con espacios amplios - como Europa o el mundoantes que con espacios más cercanos, demasiado materialistas para ellos. Dado que, según esta teoría, la extensión de los valores postmaterialistas se relaciona con el desarrollo económico, cabe esperar que la población postmaterialista aumente con el tiempo y, por ello, que los espacios geográficos más reducidos disminuyan su relevancia frente a los territorios más amplios, tendencia más visible entre los jóvenes.

En resumen, la versión de la teoría de la modernización con la que aquí se trabaja espera que en las sociedades más modernas las categorías sociales de origen y de pertenencia tengan cada vez menor capacidad para explicar las actitudes y los comportamientos de unos ciudadanos cada vez más individualistas en una sociedad cada vez más individualizada (Gobernado, 1996: 8). Para comprobar si Andalucía se está modernizando, se ha realizado una doble comparación: por un lado, se han comparado los datos de 1995 con los de 2006 para ver la evolución de la comunidad a lo largo de la década; por otro lado, se ha comparado Andalucía con Cataluña, una región con mayor grado de desarrollo económico y social, para contrastar si Andalucía se acerca a ella. Se quiere comprobar si: 1) las variables explican menos en Cataluña que en Andalucía, y 2) si en ambas comunidades lo hacen en menor medida en 2006 que en 1995.

\section{Metodología}

Se ha empleado como base de datos para los años 1995 y 2006 los sondeos que realiza mensualmente la empresa ASEP. Cada encuesta se realiza sobre una muestra representativa de la población española mayor de 16 años (1.200 entrevistas). Todos los sondeos tienen un bloque de preguntas comunes, en el que se incluyen las variables que son objeto de análisis en este estudio. El diseño es tal que permite la adición de los resultados de los bloques fijos de preguntas de las sucesivas encuestas. De esta manera, para el año 1995, la muestra de Cataluña tiene 2.094 casos y la de Andalucía, 2.385, mientras que en 2006 ascienden a 2.123 y 2.297, respectivamente. Para asegurar la representatividad de la muestra, se han buscado fuentes de datos adicionales (INE, CIS, etc.) de las principales variables para comprobar su coincidencia con la distribución de los casos en la base de ASEP.

La variable «sentimiento nacionalista» se recoge en la pregunta: «En general, ¿diría Vd. que se siente más (extremeño, catalán, gallego, etc.) que español, tan (canario, etc.) como español, o más español que (valenciano, vasco, etc.)? Sólo se siente (vasco, gallego, etc. / Más (vasco, etc.) que español / Tan (vasco, etc.) como español / Más español que (vasco, etc.) / Sólo se siente español / No sabe / No contesta». Sin embargo, en este trabajo se ha preferido utilizar una variable que recodifica en tres grupos a los encuestados: aquellos que contestan que se sienten exclusivamente nacionalistas o más nacionalistas que españoles se han agrupado en la categoría «nacionalista», las personas que decla- 
ran sentirse tan de un sitio como de otro se clasifican en la categoría «identidad dual» y los que declaran ser exclusivamente españolistas o más españolistas que de su comunidad se incluyen en la categoría «españolista». De esta manera, se simplifica la comprensión de las tablas, aunque se pierda parte de la riqueza de la variable original.

La variable "primer espacio con el que se siente identificado» se formula de la siguiente forma: «En otro orden de cosas, todos nos sentimos más ligados a unos grupos que a otros. Concretamente, y de esta lista de espacios geográficos, ¿con cuál se siente más identificado? Es decir, se siente Vd. sobre todo ciudadano de: Su pueblo o ciudad / Su provincia / Su comunidad autónoma / España / Europa / Occidente / El mundo / Ninguno / No sabe / No contesta».

En cuanto al resto de variables, se han empleado 1) el sexo; 2) la edad (agrupada en cuatro categorías: de 18 a 34 años, de 35 a 44, de 45 a 64 y de 65 y más años); 3) el nivel educativo (clasificado en "sin estudios, estudios primarios, estudios secundarios y estudios superiores»); 4) la ideología (las siete categorías originales han sido reagrupadas en tres: izquierda, centro y derecha); 5) el índice de valores postmaterialistas (construido por los propios investigadores de ASEP con la respuesta a dos preguntas sobre cuáles son los objetivos más relevantes para el país); 6) una adaptación del índice de posición social de Galtung (1964) (también elaborado por los investigadores de ASEP teniendo en cuenta sexo, edad, ocupación, educación, provincia de residencia, hábitat de residencia y nivel de ingresos en el hogar); 7) el estatus ocupacional, y 8) el tamaño del hábitat (que se clasifica en rural —menos de 10.000 habitantes-, semiurbano — de 10.000 a 100.000 habitantes — y urbano — más de 100.000 habitantes-). También se han comprobado las relaciones entre el sentimiento nacionalista y el espacio geográfico con el que se siente más identificado.

Puesto que las variables mencionadas tienen un carácter cualitativo, se han analizado mediante tablas de contingencia, a las que se ha realizado la prueba de significación estadística chi-cuadrado y se le ha calculado el coeficiente de contingencia. Se prestará atención a las casillas cuya frecuencia esté por debajo o por encima de lo esperable al $\mathrm{azar}^{2}$. Por motivos de espacio, en las ocasiones en que una variable resulta significativa para una sola de las comunidades en un determinado año, se comentarán brevemente los aspectos más relevantes en ella, pero no se incluirán los datos en las tablas.

El espacio geográfico con el que se siente identificado en primer lugar sólo está disponible para el año 2006, por lo que únicamente se compararán Andalucía y Cataluña para ver si, efectivamente, las variables tienen menor capacidad explicativa en la segunda que en la primera. En cuanto al sentimiento nacionalista, al estar disponibles los datos de 1995 y 2006, se realizará la doble comparación, temporal y espacial, que se ha comentado anteriormente. Por ello, comenzaremos por el espacio geográfico, para pasar después al

2. En general, no se comentarán los resultados cuando el cruce de dos categorías tenga menos de veinte casos, dado que es arriesgado extraer conclusiones con tan pocos datos. 
Tabla 1. Distribución del espacio geográfico con el que se siente más identificado y del sentimiento nacionalista, en porcentajes

\begin{tabular}{lcc}
\hline Espacio geográfico & Andalucía & Cataluña \\
\hline 2006 & & \\
Su pueblo o ciudad & 48,3 & 2,8 \\
Su provincia & 9,5 & 0,7 \\
Su comunidad autónoma & 15,5 & 40,4 \\
España & 23,5 & 48,3 \\
Europa & 1,0 & 2,4 \\
Occidente & 0,1 & 0,1 \\
El mundo & 1,8 & 5,1 \\
Ninguno & 0,4 & 0,2 \\
\hline Total (N) & $100(2.072)$ & $100(1.923)$ \\
\hline Sentimiento nacionalista & Andalucía & Cataluña \\
\hline 1995 & & \\
Nacionalista & 23,1 & 30 \\
Identidad dual & 64 & 45,6 \\
Españolista & 12,9 & 24,4 \\
\hline Total (N) & $100(2.365)$ & $100(2.066)$ \\
\hline 2006 & & \\
Nacionalista & 13,9 & 30,1 \\
Identidad dual & 71,5 & 54,5 \\
Españolista & 14,6 & 15,4 \\
\hline Total (N) & $100(2.242)$ & $100(2.100)$ \\
\hline Funte: ASEP & &
\end{tabular}

Fuente: ASEP y elaboración propia.

sentimiento nacionalista. Pero, antes de ello, se incluye en la tabla 1 la distribución de las dos variables dependientes en Andalucía y Cataluña.

Se observa que la distribución de las categorías es muy diversa en ambas comunidades: mientras que casi la mitad de los andaluces identifican como primer territorio su pueblo o ciudad, el mismo porcentaje de los catalanes eligen España, un resultado sorprendente a primera vista, dado que el sentimiento españolista es mucho más minoritario en esta comunidad de lo que pudiera pensarse viendo esta distribución, como se comprueba en la parte inferior de la tabla. Una explicación es que en la muestra exista un alto número de personas procedentes de otras regiones españolas que residen desde hace tiempo en Cataluña, por lo que podrían sentirse, por un lado, desligadas de su lugar de origen, pero, por otro, no escoger su lugar de residencia como el primer espacio con el que identificarse, sino España, que abarca a ambos territorios en sus fronteras. Para corroborar esta hipótesis, se ha comprobado que el $64,1 \%$ de los encuestados nació en la comunidad, el 33,5\% en otra región y el 2,4\% en otro país. Por ello, resulta plausible esta explicación, aunque no sea completa, dado que la cifra de los que eligen España es quince puntos porcentuales superior. 
En Cataluña, el segundo espacio más mencionado es la comunidad autónoma $(40,4 \%)$. El resto de territorios tienen un porcentaje anecdótico, salvo el mundo, categoría que congrega al 5,1\% de los encuestados. En Andalucía, el segundo territorio es España (casi la cuarta parte de la población) y, en tercer lugar, la comunidad (15,5\%), mientras que el resto de opciones son minoritarias, salvo la provincia, que es elegida por casi uno de cada diez encuestados. Podemos concluir que, en general, los catalanes, que viven en una sociedad más moderna, se identifican con territorios más amplios que los andaluces, tal y como espera la hipótesis de Inglehart, aunque pueden estar influyendo otro tipo de variables, como los flujos migratorios.

Por lo que respecta a la distribución del sentimiento nacionalista, en 1995 los nacionalistas y españolistas son más numerosos en Cataluña que en Andalucía, en detrimento de las personas con identidad dual. En 2006, los porcentajes de españolistas son similares, en ambas aumenta la población con identidad dual y se incrementan las distancias de la población nacionalista catalana frente a la andaluza, puesto que la primera dobla la cifra de la segunda ${ }^{3}$.

\section{Comparación del espacio con el que se siente más identificado (2006)}

La tabla 2 muestra cómo se relacionan el nivel educativo y los valores postmaterialistas con el espacio escogido. El nivel educativo es relevante en ambas comunidades, y en mayor medida en Andalucía ${ }^{4}$. En esta última, existe una asociación positiva entre un mayor nivel educativo y la elección de espacios geográficos amplios, alejados de la experiencia más cotidiana y cercana. Cataluña se distingue por una mayor elección de España por parte de personas sin estudios o con estudios primarios y una menor preferencia de este territorio de quienes tienen estudios secundarios, mientras que la tendencia de los universitarios es similar a la andaluza. Por otro lado, se espera que los valores postmaterialistas se relacionen de manera positiva con el espacio de pertenencia y, efectivamente, este modelo es significativo para las dos comunidades, aunque en mayor medida en Cataluña que en Andalucía. Ello puede deberse a que la

3. El análisis de la variable original utilizada por ASEP (cinco categorías) muestra que, en 1995, los que se consideran exclusivamente nacionalistas son el triple en Cataluña que en Andalucía, mientras que la cifra es similar en aquellos que se consideran más nacionalistas que españoles (alrededor del 20\%). Por último, los españolistas (exclusivos o no) son más numerosos en Cataluña que en Andalucía, tal vez porque gran parte de las personas que responden a este perfil son inmigrantes de otras comunidades. Once años después, se observa que la identificación exclusivamente nacionalista ha decaído en ambas comunidades, pero la «más nacionalista» ha bajado en Andalucía y ha subido en Cataluña. Los españolistas han disminuido su relevancia numérica en Cataluña, mientras que en Andalucía casi se duplican los exclusivamente españolistas, a la vez que disminuye el porcentaje de los más españolistas, puede que porque se hayan radicalizado las posturas políticas.

4. Hay que tener en cuenta que la distribución de la población según su nivel de estudios es bastante diferente en ambas comunidades, con una mayor presencia de gente con estudios menores de secundarios en Andalucía $(67,1 \%)$ frente a Cataluña $(52,8 \%)$, como se puede observar en la fila que recoge los totales de la columna. 

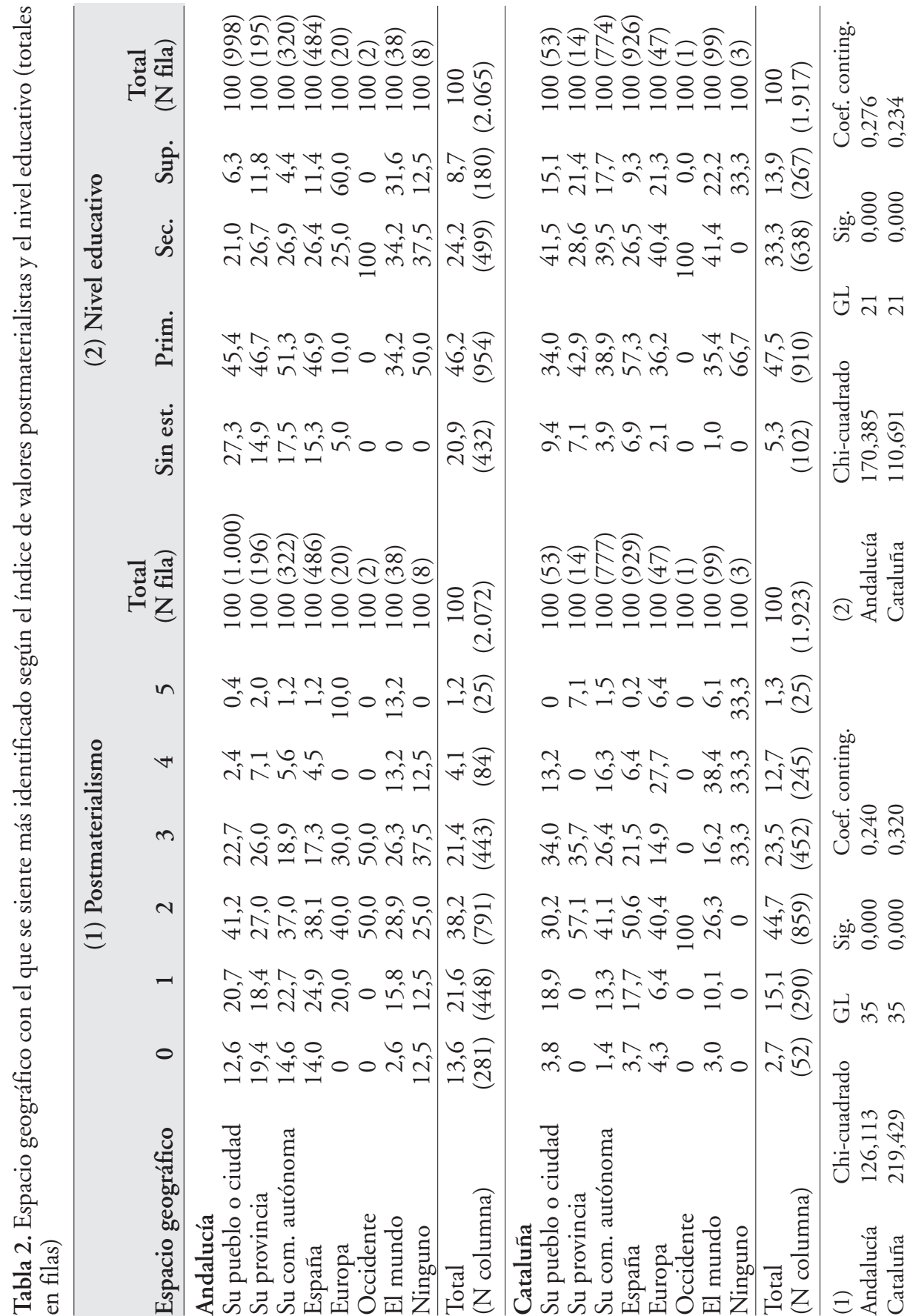

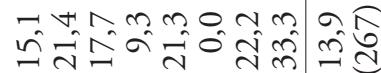

n $n$ n

nतn naอmm

ॠ

Hnma no nO जैच

manno

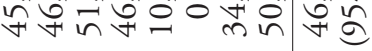

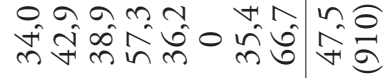

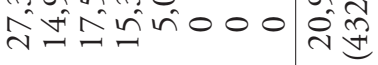

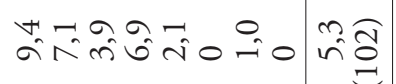

$\frac{8}{\pi}$

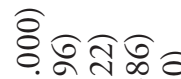

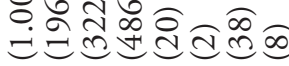

ㅇㅇㅇㅇㅇㅇㅇㅇ

$8 \widehat{ก}$

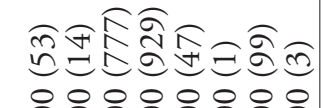

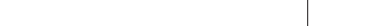

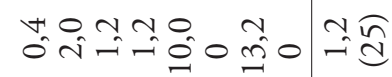

으응ㅇㅇㅇㅇㅇㅇㅇㅇㅇㅡ.

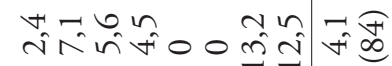

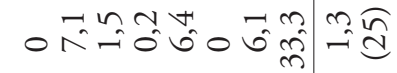

r.

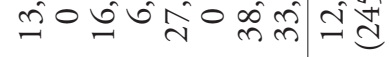

nogmoomn

สิ่

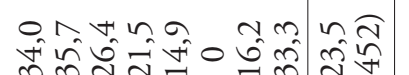

원

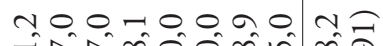

r

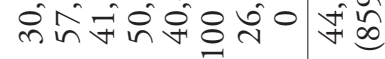

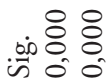

HNOO

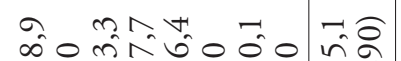

ำกำำำำ

6roo 6 n

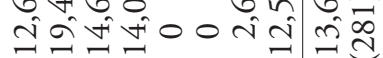

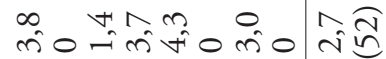

تิn

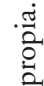

苂

苞

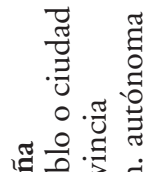

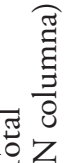

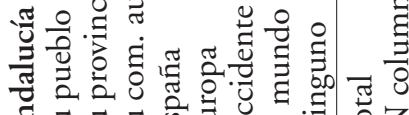

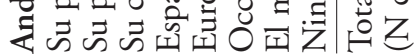

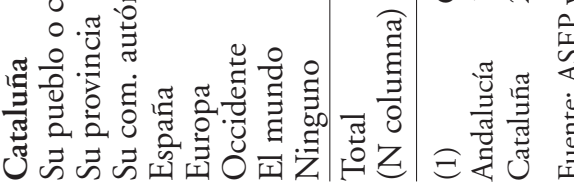


población postmaterialista es mucho mayor en esta segunda comunidad (para un análisis detallado, ver, en este mismo número, el artículo de Fernández Alonso «Valores y creencias en el proceso de modernización»). En todo caso, la pauta que se encuentra es similar en ambas comunidades en lo que a la población más postmaterialista se refiere y se confirma la hipótesis de Inglehart, pues eligen con mayor frecuencia Europa y el mundo. En cambio, se encuentran más diferencias entre los materialistas: los andaluces eligen más la provincia, la región y España, mientras que los catalanes eligen más el pueblo o la ciudad y España ${ }^{5}$. Los casos mixtos en Cataluña tienden a escoger más la provincia y España y la localidad, la provincia y la región, mientras que en Andalucía se decantan por el pueblo o la ciudad y por Europa.

La tabla 3 muestra la relación entre el espacio geográfico y la posición social. Esta tabla de contingencia es especialmente significativa en el caso andaluz. En principio, dado que este índice se relaciona, entre otros factores, con el nivel educativo y el tamaño del hábitat, es esperable que las personas con posición social más alta tiendan a elegir espacios más amplios. Efectivamente, se puede observar que, a medida que se avanza por el índice, los territorios más mencionados pasan de ser el pueblo o la ciudad y la provincia a la comunidad autónoma y España en posiciones intermedias, y Europa y el mundo en posiciones más altas.

El estatus ocupacional (tabla 4) es también una variable relevante para estudiar el espacio geográfico, con una chi-cuadrado ligeramente superior en Cataluña. La muestra andaluza posee más personas en paro, amas de casa y estudiantes, y menos jubilados, que la muestra catalana. Ello se corresponde con la estructura del mercado laboral en ambas comunidades, excepto en lo que se refiere al número de estudiantes, dado que la tasa de escolarización es menor en Andalucía. La distribución de los estatus ocupacionales alto, medio y bajo es bastante similar. Observando la tabla 4, se puede concluir que las pautas son bastante diferentes en Andalucía y Cataluña. Los andaluces que más se identifican con el pueblo o la ciudad son las personas en paro, las amas de casa y los jubilados, mientras que se identifican menos con este espacio las personas de estatus alto o medio. Estas personas, en cambio, tienden a identificarse más con la región, España e incluso Europa. El mundo es mencionado con mayor frecuencia por personas de estatus bajo, en paro y estudiantes, lo que puede justificarse por motivos de edad, ya que es probable que hayan contestado esta categoría personas jóvenes, que aún no se han posicionado en el mercado laboral. Por su parte, en Cataluña, las personas de estatus alto eligen más la provincia, Europa y el mundo, Cataluña es escogida por las de estatus medio y bajo, y su pueblo o su ciudad, por las de estatus bajo, las personas en paro, los jubilados y los estudiantes, pero no por las amas de casa. Ello podría deberse a que un porcentaje relevante de estas amas de casa sean mujeres de mediana o avanzada edad que inmigraron de otras comunidades, una hipótesis que se

5. Destaca un caso que se desvía de la teoría de Inglehart: la mayor identificación de lo esperable al azar de los materialistas catalanes con Europa y, en menor medida, el mundo. 
Tabla 3. Espacio geográfico con el que se siente más identificado según el índice de posición social de Galtung (totales en filas)

\begin{tabular}{|c|c|c|c|c|c|c|c|c|c|c|c|}
\hline \multicolumn{2}{|l|}{$\begin{array}{l}\text { Espacio } \\
\text { geográfico }\end{array}$} & $\begin{array}{c}0 . \\
\text { Muy baja }\end{array}$ & 1 & 2 & 3 & 4 & 5 & 6 & 7 & $\begin{array}{c}8 . \\
\text { Muy alta }\end{array}$ & $\begin{array}{l}\text { Total } \\
\text { (N fila) }\end{array}$ \\
\hline \multicolumn{12}{|l|}{ Andalucía } \\
\hline \multicolumn{2}{|c|}{ Su pueblo o ciudad } & 3,9 & 15,5 & 24,7 & 20,4 & 17,6 & 9,3 & 6,6 & 1,6 & 0,4 & $100(1.000)$ \\
\hline \multicolumn{2}{|l|}{ Su provincia } & 5,1 & 15,3 & 20,9 & 20,9 & 15,8 & 12,8 & 6,1 & 1,5 & 1,5 & 100 (196) \\
\hline \multicolumn{2}{|c|}{ Su com. autónoma } & 2,2 & 9,6 & 21,1 & 21,7 & 15,2 & 14,6 & 14,3 & 1,2 & 0 & $100(322)$ \\
\hline \multicolumn{2}{|l|}{ España } & 2,9 & 7,6 & 21,4 & 17,9 & 14,8 & 18,9 & 11,1 & 4,3 & 1,0 & $100(486)$ \\
\hline \multicolumn{2}{|l|}{ Europa } & 5,0 & 0 & 10,0 & 5,0 & 5,0 & 10,0 & 35,0 & 10,0 & 20,0 & $100(20)$ \\
\hline \multicolumn{2}{|l|}{ Occidente } & 0 & 0 & 0 & 50,0 & 0 & 50,0 & 0 & 0 & 0 & $100(2)$ \\
\hline \multicolumn{2}{|l|}{ El mundo } & 0 & 7,9 & 13,2 & 15,8 & 13,2 & 21,1 & 18,4 & 2,6 & 7,9 & $100(38)$ \\
\hline \multicolumn{2}{|l|}{ Ninguno } & 0 & 0 & 25,0 & 25,0 & 0 & 25,0 & 25,0 & 0 & 0 & $100(8)$ \\
\hline \multicolumn{2}{|l|}{$\begin{array}{l}\text { Total } \\
\text { (N columna) }\end{array}$} & $\begin{array}{r}3,4 \\
(71)\end{array}$ & $\begin{array}{r}12,4 \\
(256)\end{array}$ & $\begin{array}{r}22,6 \\
(469)\end{array}$ & $\begin{array}{r}19,9 \\
(412)\end{array}$ & $\begin{array}{c}16,1 \\
(334)\end{array}$ & $\begin{array}{r}13,0 \\
(270)\end{array}$ & $\begin{array}{r}9,4 \\
(194)\end{array}$ & $\begin{array}{r}2,3 \\
(194)\end{array}$ & $\begin{array}{r}0,9 \\
(47)\end{array}$ & $\begin{array}{r}100 \\
(2.072)\end{array}$ \\
\hline \multicolumn{12}{|l|}{ Cataluña } \\
\hline \multicolumn{2}{|c|}{ Su pueblo o ciudad } & 3,8 & 9,4 & 11,3 & 35,8 & 17,0 & 11,3 & 7,5 & 3,8 & 0 & $100(53)$ \\
\hline \multicolumn{2}{|c|}{ Su provincia } & 7,1 & 7,1 & 14,3 & 42,9 & 7,1 & 0 & 0 & 21,4 & 0 & $100(14)$ \\
\hline \multicolumn{2}{|c|}{ Su com. autónoma } & 1,8 & 9,5 & 20,3 & 19,3 & 16,7 & 14,9 & 11,2 & 6,0 & 0,1 & $100(777)$ \\
\hline \multicolumn{2}{|c|}{ España } & 1,2 & 6,4 & 20,7 & 22,8 & 21,6 & 16,8 & 7,6 & 2,5 & 0,4 & $100(929)$ \\
\hline \multicolumn{2}{|l|}{ Europa } & 0 & 2,1 & 14,9 & 23,4 & 25,5 & 14,9 & 14,9 & 4,3 & 0 & $100(47)$ \\
\hline \multicolumn{2}{|l|}{ Occidente } & 0 & 0 & 0 & 0 & 100 & 0 & 0 & 0 & 0 & $100(1)$ \\
\hline \multicolumn{2}{|l|}{ El mundo } & 0 & 5,1 & 11,1 & 19,2 & 19,2 & 21,2 & 14,1 & 10,1 & 0 & $100(99)$ \\
\hline \multicolumn{2}{|l|}{ Ninguno } & 0 & 0 & 0 & 0 & 0 & 66,7 & 33,3 & 0 & 0 & $100(3)$ \\
\hline \multirow{3}{*}{$\begin{array}{l}\text { Total } \\
\text { (N columna) }\end{array}$} & & $<$ & 7,5 & 19,6 & 21,7 & 19,4 & 16,0 & 9,6 & 4,5 & 0,3 & 100 \\
\hline & & (28) & (145) & (376) & $(417)$ & (373) & (308) & (184) & (87) & (5) & (1.923) \\
\hline & \multicolumn{2}{|c|}{ Chi-cuadrado } & GL & & Sig. & & \multicolumn{2}{|c|}{ Coef. conting. } & & & \\
\hline \multirow{2}{*}{$\begin{array}{l}\text { Andalucía } \\
\text { Cataluña }\end{array}$} & \multicolumn{2}{|c|}{241,448} & 56 & & 0,000 & & \multicolumn{2}{|c|}{0,323} & & & \\
\hline & \multicolumn{2}{|c|}{102,004} & 56 & & 0,000 & & 0,224 & & & & \\
\hline
\end{tabular}

Fuente: ASEP y elaboración propia.

apoya en el hecho de que estas amas de casa tampoco eligen frecuentemente la provincia ni Cataluña como espacios relevantes, sino España.

La última tabla de este apartado (tabla 5) muestra la relación entre el espacio escogido y la ideología, y entre éste y el sentimiento nacionalista. Este último análisis nos sirve de transición al siguiente epígrafe, en el que analizaremos qué variables explican mejor el sentimiento nacionalista. Podemos observar que la ideología es un elemento relevante para explicar el espacio con que se siente identificado el individuo, especialmente en el caso andaluz. Hay que tener en cuenta que, en ambas regiones, la población se declara mayoritariamente de izquierdas, especialmente en Cataluña (tres cuartas partes) y muy pocos optan por la derecha (en el caso catalán, el 3,7\%). En Andalucía, las personas que se autoposicionan como de izquierdas eligen los espacios geográficos más próximos, aunque también existe un colectivo, más minoritario, que escoge Europa y el 
Tabla 4. Espacio geográfico con el que se siente más identificado según el estatus ocupacional (totales en filas, 2006)

\begin{tabular}{|c|c|c|c|c|c|c|c|c|c|c|}
\hline \multirow{2}{*}{\multicolumn{2}{|c|}{$\begin{array}{l}\text { Espacio } \\
\text { geográfico }\end{array}$}} & \multicolumn{8}{|c|}{ Estatus ocupacional } & \multirow[b]{2}{*}{$\begin{array}{l}\text { Total } \\
\text { (N fila) }\end{array}$} \\
\hline & & Alto & Medio & Bajo & $\begin{array}{l}\text { En } \\
\text { paro }\end{array}$ & $\begin{array}{c}\text { Ama } \\
\text { de casa }\end{array}$ & Jubilado & Estudiante & Otros & \\
\hline \multicolumn{11}{|l|}{ Andalucía } \\
\hline \multicolumn{2}{|c|}{ Su pueblo o ciudad } & 3,1 & 20,5 & 17,8 & 11,9 & 22,4 & 18,2 & 5,8 & 0,2 & $100(994)$ \\
\hline \multicolumn{2}{|c|}{ Su provincia } & 5,7 & 32,8 & 11,5 & 8,3 & 19,3 & 13,5 & 8,3 & 0,5 & $100(192)$ \\
\hline \multicolumn{2}{|c|}{ Su com. autónoma } & 6,5 & 28,9 & 21,4 & 5,3 & 18,3 & 13,0 & 6,2 & 0,3 & $100(322)$ \\
\hline \multicolumn{2}{|l|}{ España } & 9,3 & 29,0 & 17,4 & 4,3 & 18,4 & 15,7 & 5,8 & 0 & $100(483)$ \\
\hline \multicolumn{2}{|l|}{ Europa } & 25,0 & 35,0 & 15,0 & 0 & 5,0 & 20,0 & 0 & 0 & $100(20)$ \\
\hline \multicolumn{2}{|l|}{ Occidente } & 0 & 0 & 0 & 50,0 & 0 & 0 & 50,0 & 0 & $100(2)$ \\
\hline \multicolumn{2}{|l|}{ El mundo } & 2,6 & 31,6 & 21,1 & 10,5 & 5,3 & 13,2 & 15,8 & 0 & $100(38)$ \\
\hline \multicolumn{2}{|l|}{ Ninguno } & 0 & 25,0 & 37,5 & 25,0 & 0 & 12,5 & 0 & 0 & $100(8)$ \\
\hline \multicolumn{2}{|l|}{$\begin{array}{l}\text { Total } \\
\text { (N columna) }\end{array}$} & $\begin{array}{r}5,5 \\
(114)\end{array}$ & $\begin{array}{r}25,3 \\
(521)\end{array}$ & $\begin{array}{r}17,8 \\
(366)\end{array}$ & $\begin{array}{c}8,7 \\
(179)\end{array}$ & $\begin{array}{c}20,0 \\
(411)\end{array}$ & $\begin{array}{r}16,3 \\
(335)\end{array}$ & $\begin{array}{r}6,3 \\
(129)\end{array}$ & $\begin{array}{l}0,2 \\
(4)\end{array}$ & $\begin{array}{r}100 \\
(2.059)\end{array}$ \\
\hline \multicolumn{11}{|l|}{ Cataluña } \\
\hline \multicolumn{2}{|c|}{ Su pueblo o ciudad } & 5,7 & 20,8 & 30,2 & 7,5 & 7,5 & 22,6 & 5,7 & 0 & $100(53)$ \\
\hline \multicolumn{2}{|l|}{ Su provincia } & 21,4 & 7,1 & 21,4 & 0 & 14,3 & 28,6 & 7,1 & 0 & $100(14)$ \\
\hline \multicolumn{2}{|c|}{ Su com. autónoma } & 8,2 & 32,4 & 11,7 & 3,0 & 15,2 & 22,3 & 7,1 & 0,3 & $100(772)$ \\
\hline \multicolumn{2}{|l|}{ España } & 4,6 & 25,5 & 22,0 & 6,3 & 16,4 & 22,2 & 2,5 & 0,5 & $100(928)$ \\
\hline \multicolumn{2}{|l|}{ Europa } & 12,8 & 27,7 & 12,8 & 8,5 & 6,4 & 21,3 & 10,6 & 0 & $100(47)$ \\
\hline \multicolumn{2}{|l|}{ Occidente } & 0 & 0 & 0 & 0 & 0 & 0 & 100 & 0 & $100(1)$ \\
\hline \multicolumn{2}{|l|}{ El mundo } & 15,3 & 26,5 & 23,5 & 5,1 & 11,2 & 10,2 & 8,2 & 0 & $100(98)$ \\
\hline \multicolumn{2}{|l|}{ Ninguno } & 66,7 & 0 & 33,3 & 0 & 0 & 0 & 0 & 0 & $100(3)$ \\
\hline \multirow[t]{2}{*}{$\begin{array}{l}\text { Total } \\
\text { (N columna) }\end{array}$} & & $\begin{array}{r}7,0 \\
(135)\end{array}$ & $\begin{array}{r}28,1 \\
(538)\end{array}$ & $\begin{array}{r}17,9 \\
(343)\end{array}$ & $\begin{array}{l}4,9 \\
(94)\end{array}$ & $\begin{array}{l}15,1 \\
(289)\end{array}$ & $\begin{array}{r}21,6 \\
(414)\end{array}$ & $\begin{array}{r}5,0 \\
(96)\end{array}$ & $\begin{array}{l}0,4 \\
(7)\end{array}$ & $\begin{array}{r}100 \\
(1.916)\end{array}$ \\
\hline & \multicolumn{2}{|c|}{ Chi-cuadrado } & \multicolumn{2}{|l|}{ GL } & Sig. & \multicolumn{2}{|c|}{ Coef. conting. } & & & \\
\hline \multirow{2}{*}{$\begin{array}{l}\text { Andalucía } \\
\text { Cataluña }\end{array}$} & \multicolumn{2}{|c|}{137,550} & 49 & & 0,000 & \multicolumn{2}{|c|}{0,250} & & & \\
\hline & \multicolumn{2}{|c|}{155,099} & 49 & & 0,000 & 0,27 & & & & \\
\hline
\end{tabular}

Fuente: ASEP y elaboración propia.

mundo. En cambio, las personas de centro y derecha se decantan por España. En Cataluña, la pauta es muy similar, salvo una excepción: la provincia es preferida en mayor medida por la gente de centro y derecha que por la de izquierdas.

Por otro lado, el sentimiento nacionalista es, con diferencia, la variable con mayor chi-cuadrado y coeficiente de contingencia en el caso catalán y es superado, por muy poco, por el índice de posición social en el caso andaluz $(228,512$ frente a 241,448). Ello se debe a que, como hemos comentado, la identificación con un territorio guarda relación con la identificación con una comunidad política. Probablemente, el hecho de que el nacionalismo catalán sea más antiguo y haya arraigado más en la población que el nacionalismo andaluz justifica que esta variable sea mucho más relevante en Cataluña. En Andalucía, las personas que se sienten nacionalistas (que son prácticamente la mitad que 
Tabla 5. Espacio geográfico con el que se siente más identificado según la ideología y el sentimiento nacionalista (totales en filas, 2006)

\begin{tabular}{|c|c|c|c|c|c|c|c|c|c|}
\hline \multirow{2}{*}{$\begin{array}{l}\text { Espacio } \\
\text { geográfico }\end{array}$} & & \multicolumn{4}{|c|}{ (1) Ideología } & \multicolumn{4}{|c|}{ (2) Sentimiento nacionalista } \\
\hline & & \multirow[t]{2}{*}{ Izq. } & \multirow[t]{2}{*}{ Centro } & \multirow[t]{2}{*}{ Derecha } & $\begin{array}{c}\text { Total } \\
\text { (N fila) }\end{array}$ & \multirow[t]{2}{*}{ Nacion. } & \multirow[t]{2}{*}{ Dual } & \multirow[t]{2}{*}{ Español } & $\begin{array}{c}\text { Total } \\
\text { (N fila) }\end{array}$ \\
\hline \multicolumn{4}{|l|}{ Andalucía } & & & & & & \\
\hline \multicolumn{2}{|c|}{ Su pueblo o ciudad } & 66,7 & 19,8 & 13,6 & $100(693$ & 17,0 & 78,6 & 4,5 & $100(984)$ \\
\hline \multicolumn{2}{|l|}{ Su provincia } & 63,5 & 22,4 & 14,1 & $100(156$ & 20,0 & 56,9 & 23,1 & $100(195)$ \\
\hline \multicolumn{2}{|c|}{ Su com. autónoma } & 74,4 & 16,3 & 9,3 & $100(246$ & 18,7 & 63,5 & 17,8 & $100(315)$ \\
\hline \multicolumn{2}{|l|}{ España } & 38,2 & 35,5 & 26,3 & $100(411$ & 4,4 & 65,5 & 30,1 & $100(478)$ \\
\hline \multicolumn{2}{|l|}{ Europa } & 80,0 & 6,7 & 13,3 & $100(15)$ & 11,1 & 61,1 & 27,8 & $100(18)$ \\
\hline \multicolumn{2}{|l|}{ Occidente } & 0 & 100 & 0 & $100(1)$ & 50,0 & 50,0 & 0 & $100(2)$ \\
\hline \multicolumn{2}{|l|}{ El mundo } & 87,1 & 12,9 & 0 & $100(31)$ & 7,7 & 69,2 & 23,1 & $100(26)$ \\
\hline \multicolumn{2}{|l|}{ Ninguno } & 80,0 & 0 & 20,0 & $100(5)$ & 0 & 66,7 & 33,3 & $100(3)$ \\
\hline \multicolumn{2}{|l|}{$\begin{array}{l}\text { Total } \\
\text { (N columna) }\end{array}$} & $\begin{array}{r}60,6 \\
(944)\end{array}$ & $\begin{array}{l}23,4 \\
(364)\end{array}$ & $\begin{array}{r}16,0 \\
(250)\end{array}$ & $\begin{array}{c}100 \\
(1.558)\end{array}$ & $\begin{array}{l}14,4 \\
(291)\end{array}$ & $\begin{array}{c}70,7 \\
(1.429)\end{array}$ & $\begin{array}{l}14,9 \\
(301)\end{array}$ & $\begin{array}{r}100 \\
(2.021)\end{array}$ \\
\hline \multicolumn{10}{|l|}{ Cataluña } \\
\hline \multicolumn{2}{|c|}{ Su pueblo o ciudad } & 78,8 & 18,2 & 3,0 & $100(33)$ & 54,7 & 37,7 & 7,5 & $100(53)$ \\
\hline \multicolumn{2}{|l|}{ Su provincia } & 60,0 & 30,0 & 10,0 & $100(10)$ & 28,6 & 50,0 & 21,4 & $100(14)$ \\
\hline \multicolumn{2}{|c|}{ Su com. autónoma } & 77,2 & 21,3 & 1,5 & $100(657$ & 61,9 & 35,9 & 2,2 & $100(767)$ \\
\hline \multicolumn{2}{|c|}{ España } & 73,0 & 21,4 & 5,6 & $100(766$ & 3,9 & 69,5 & 26,6 & $100(929)$ \\
\hline \multicolumn{2}{|l|}{ Europa } & 79,5 & 15,9 & 4,5 & $100(44)$ & 27,7 & 53,2 & 19,1 & $100(47)$ \\
\hline \multicolumn{2}{|l|}{ Occidente } & 0,0 & 100 & 0 & $100(1)$ & 0 & 0 & 100,0 & $100(1)$ \\
\hline \multicolumn{2}{|l|}{ El mundo } & 91,4 & 7,4 & 1,2 & $100(81)$ & 24,7 & 59,6 & 15,7 & $100(89)$ \\
\hline \multicolumn{2}{|l|}{ Ninguno } & 66,7 & 0 & 33,3 & $100(3)$ & 0 & 100 & 0 & $100(3)$ \\
\hline $\begin{array}{l}\text { Total } \\
\text { (N columna) }\end{array}$ & & $\begin{array}{r}75,8 \\
(1.209)\end{array}$ & $\begin{array}{l}20,5 \\
(327)\end{array}$ & $\begin{array}{r}3,7 \\
(59)\end{array}$ & $\begin{array}{c}100 \\
(1.595)\end{array}$ & $\begin{array}{l}30,4 \\
(579)\end{array}$ & $\begin{array}{r}54,1 \\
(1.029)\end{array}$ & $\begin{array}{l}15,5 \\
(295)\end{array}$ & $\begin{array}{r}100 \\
(1.903)\end{array}$ \\
\hline (1) & Chi- & -cuadrado & GL & Sig. & & oef. cor & & & \\
\hline Andalucía & 135 & ,722 & 14 & 0,00 & 00 & 0,283 & & & \\
\hline Cataluña & 42,4 & 459 & 14 & 0,00 & 00 & 0,161 & & & \\
\hline (2) & Chi-c & -cuadrado & GL & Sig. & & Coef. conting. & & & \\
\hline Andalucía & 228 &, 512 & 14 & 0,00 & 00 & 0,319 & & & \\
\hline Cataluña & 739 & ,998 & 14 & 0,00 & 00 & 0,529 & & & \\
\hline
\end{tabular}

Fuente: ASEP y elaboración propia.

en Cataluña), tienden a identificarse más con los ámbitos más cercanos (su pueblo o su ciudad, su provincia y su comunidad), las personas con una identificación dual eligen en mayor medida su localidad de residencia (por tanto, no escogen ni España ni Andalucía, tras haber declarado que se sienten parte tanto de un ámbito como de otro) y las personas españolistas eligen la provincia, España y, curiosamente, Andalucía, como sus territorios más destacados. En el caso catalán, encontramos algunas pautas similares y otras diferentes. De nuevo, las personas que se consideran nacionalistas eligen en mayor medida el pueblo o la ciudad y la región, pero no la provincia. En cambio, las personas 
con una identificación dual escogen España en mayor medida que otros espacios y, también, el mundo. Los españolistas, por último, escogen, además de a España, a la provincia y a Europa como sus espacios más relevantes ${ }^{6}$.

\section{Evolución y comparación del sentimiento nacionalista (1995 y 2006)}

La primera tabla de contingencia de este apartado (tabla 6) recoge el cruce de la variable «sentimiento nacionalista» con la edad, en primer lugar, y con el nivel educativo, en segundo lugar ${ }^{7}$. El modelo no es significativo según la edad para Andalucía en 1995, por lo que no se incluye. En ese año, la población catalana más joven se identificaba más como nacionalista, mientras que la población de las dos categorías intermedias mantenían una identidad dual o bien un sentimiento españolista; por último, los ancianos elegían tanto el nacionalismo como el españolismo. En 2006, el modelo es relevante en las dos comunidades, aunque en mayor medida en el caso catalán. Los nacionalistas andaluces son menores de 44 años, mientras que, en Cataluña, el perfil ha cambiado, pues, junto a los mayores de 65 años, se encuentra la población de 35 a 44 años. La identificación dual es más frecuente en Andalucía en todos los grupos salvo en los jóvenes, mientras que en Cataluña es más frecuente en todos los colectivos salvo los ancianos (por lo que las personas de 35 a 64 años en ambas tienden a identificarse tanto como españoles como de su comunidad). Por último, los españolistas en Andalucía son las personas de 45 a 64 años y, en menor medida, los jóvenes de 18 a 34, mientras en Cataluña se concentra en la población mayor de 45 años.

El nivel educativo muestra una relación importante con el sentimiento nacionalista en ambos años, especialmente en el caso catalán, aunque cabe resaltar que esta variable ha disminuido su importancia a lo largo de la década en esta región, mientras que ha aumentado su relevancia en Andalucía. Además,

6. Cabe mencionar que el sexo, la edad y el hábitat han sido significativos únicamente en Cataluña, por lo que no se han incluido en las tablas. Se observa que los más jóvenes se identifican en mayor medida con territorios muy amplios, así como con la localidad más cercana. Los adultos jóvenes eligen más el pueblo o la ciudad y Europa, mientras que las edades intermedias se decantan por España. Por último, la población más anciana tiende a mencionar en mayor medida la provincia y la comunidad autónoma. En cuanto al sexo, las mujeres catalanas eligen en mayor medida la comunidad autónoma y en menor medida Europa y el mundo. Ello probablemente tenga que ver con que, en la muestra, un importante porcentaje de las mujeres son amas de casa (casi un 30\%), quienes, en principio, tienen un perfil de edad más avanzada y, probablemente, de menores estudios, una variable que se relaciona fuertemente con el tipo de espacio que se elige, como ya se ha visto anteriormente. En el caso del hábitat, las personas que residen en ámbitos rurales escogen con mayor frecuencia los espacios geográficos más cercanos, los que viven en espacios semiurbanos se dividen entre los que eligen el pueblo o la ciudad y los que escogen Europa y, por último, los que viven en un hábitat urbano eligen más España y el mundo.

7. No se incluye el análisis del sexo porque sólo ha sido significativo en un caso: Andalucía en 1995. En ese año, las mujeres tendían a ser más nacionalistas y los hombres más españolistas. La identificación dual se distribuía de manera similar en ambos sexos. 
Tabla 6. Sentimiento nacionalista según edad y nivel educativo (totales en filas)

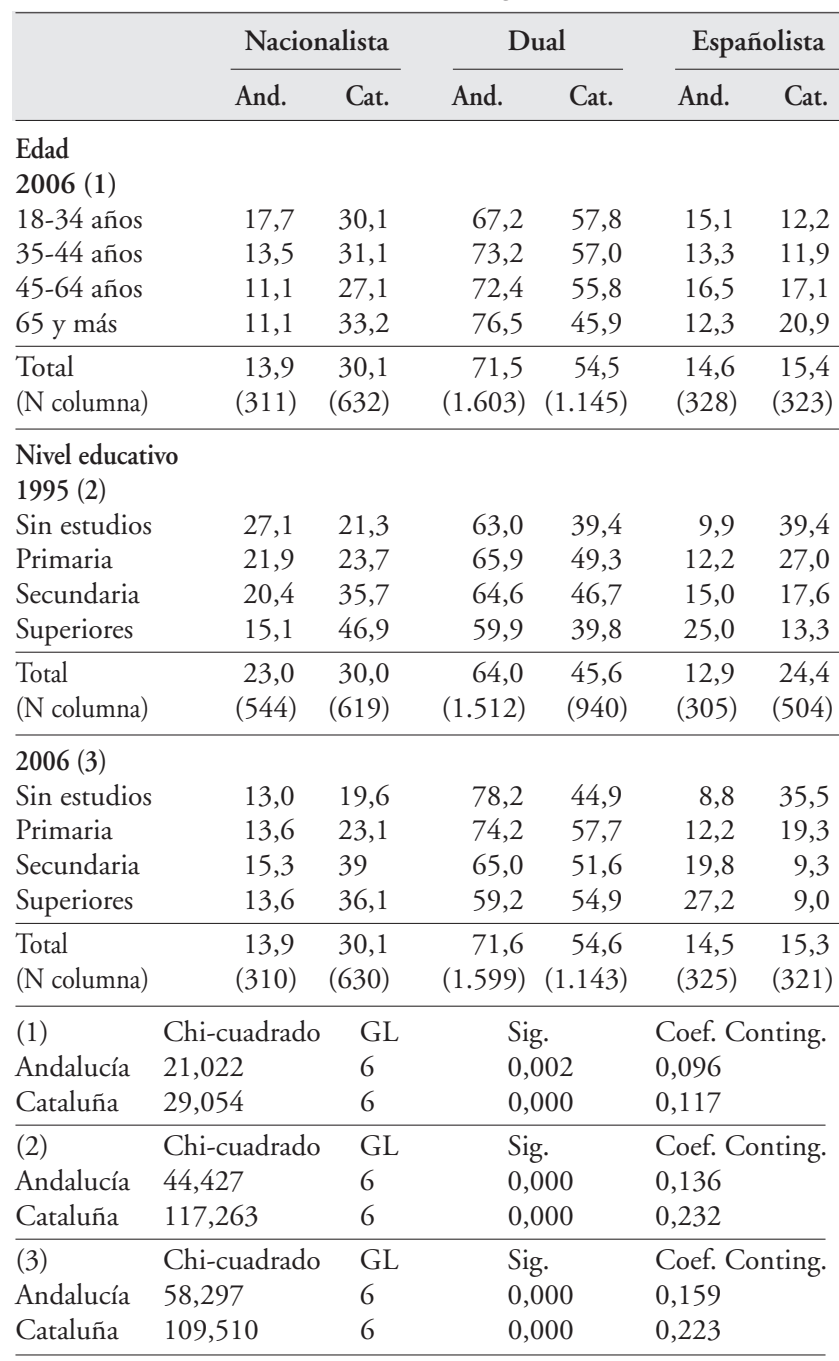

Fuente: ASEP y elaboración propia.

las pautas difieren y, probablemente, ayudan a explicar la visibilidad social que un nacionalismo y otro han tenido históricamente. En 1995, los nacionalistas andaluces se concentraban entre las personas sin estudios, mientras que los catalanes eran, sobre todo, personas universitarias y, en menor medida, con estudios secundarios. En el lado contrario, los españolistas andaluces eran más frecuentes entre las personas de las dos categorías superiores, mientras que en Cataluña se agrupaban precisamente entre las personas sin estudios y con estudios primarios. Ello puede relacionarse con el hecho de que parte de la pobla- 
ción encuestada con un bajo nivel de estudios proceda de otras comunidades, ya que, históricamente, llegaron muchas personas de extracción humilde. Las personas con identidad dual sí siguen una pauta similar en ambas comunidades, pues se concentran entre los que tienen estudios primarios y secundarios.

Once años después, el nacionalismo andaluz ha cambiado su perfil, pues ahora se concentra en las personas de estudios secundarios ( $y$ se ha visto en esta misma tabla que es gente entre 18 y 44 años), por lo que adquiere mayor protagonismo en la vida pública andaluza, donde, precisamente durante 2006, fueron numerosos los debates sobre los rasgos característicos de la nación andaluza, antes de la aprobación del nuevo Estatuto de Autonomía en febrero de 2007. En Cataluña, el perfil de los nacionalistas se mantiene igual que en 1995 , puesto que se trata de personas con estudios secundarios y superiores. Las personas con identidad dual sufren una ligera modificación con respecto a la década anterior en ambas regiones: en Andalucía, baja el nivel de estudios, pues se concentra en personas sin estudios y con estudios primarios, mientras que, en Cataluña, aumenta el nivel, pues se concentra entre los encuestados con estudios primarios y, en menor medida, con estudios superiores. Por último, los españolistas mantienen la misma pauta vista en 1995.

La tabla 7 muestra la relación entre el sentimiento nacionalista y la ideología, así como entre éste y el postmaterialismo. El modelo que recoge el índice de valores postmaterialistas no es significativo en el caso andaluz en 1995, por lo que no se incluye en la tabla. En cambio, en 2006, ambas comunidades presentan modelos significativos, aunque es mayor en el caso catalán. Las relaciones de los valores postmaterialistas con el sentimiento nacional llaman la atención por ser claramente diferentes. El materialismo se concentra entre los nacionalistas en Andalucía y entre los españolistas en Cataluña, y justo lo contrario ocurre con el postmaterialismo. La pauta catalana ya se producía en 1995, pero de manera más atenuada que una década después. Únicamente coinciden ambas comunidades en el hecho que los valores mixtos tienden a darse entre las personas que se identifican de manera dual.

Por lo que respecta a la ideología, se puede encontrar, como ocurriera con el nivel educativo en 1995, una pauta similar en ambas regiones en el caso de la identidad dual: son las personas de izquierdas las que eligen en mayor medida esa opción. En cambio, los nacionalistas andaluces se consideran de izquierdas y los catalanes, de centro. Por último, los españolistas se concentran en Andalucía en el centro y la derecha, pero en Cataluña se distribuyen entre la izquierda y la derecha, un hecho algo sorprendente, dado que el discurso de la izquierda no suele ensalzar la nación española ${ }^{8}$. En 2006, se pueden obser-

8. Es probable que la explicación se encuentre otra vez en el alto número de emigrantes, ya que una de las mayores concentraciones de votos del PSC se da en los municipios más industriales situados alrededor de Barcelona, donde la emigración fue numerosa. Sin embargo, la base de datos no recoge la provincia de nacimiento de los encuestados, con lo que no podemos contrastar si un alto porcentaje de ellos es emigrante, aunque parece razonable pensar que así sea, dado que se trata de una muestra representativa. 
Tabla 7. Sentimiento nacionalista según la ideología y el índice de valores postmaterialistas (totales en filas)

\begin{tabular}{|c|c|c|c|c|c|c|c|c|}
\hline & \multicolumn{2}{|c|}{ Nacionalista } & \multicolumn{2}{|c|}{ Dual } & \multicolumn{2}{|c|}{ Españolista } & \multicolumn{2}{|c|}{ Total (N fila) } \\
\hline & And. & Cat. & And. & Cat. & And. & Cat. & And. & Cat. \\
\hline \multicolumn{9}{|l|}{ Ideología } \\
\hline \multicolumn{9}{|l|}{1995 (1) } \\
\hline Izquierda & 26,6 & 26,0 & 64,3 & 46,7 & 9,1 & 27,3 & $100(1.020)$ & $100(935)$ \\
\hline Centro & 20,2 & 42,2 & 61,1 & 37,7 & 18,7 & 20,2 & $100(252)$ & $100(377)$ \\
\hline Derecha & 18,0 & 31,0 & 57,3 & 43,1 & 24,7 & 25,8 & $100(328)$ & $100(248)$ \\
\hline Total & 23,8 & 30,7 & 62,4 & 44,0 & 13,8 & 25,3 & 100 & 100 \\
\hline ( $\mathrm{N}$ columna) & (381) & $(479)$ & (998) & $(686)$ & $(221)$ & (395) & $(1.600)$ & $(1.560)$ \\
\hline \multicolumn{9}{|l|}{$2006(2)$} \\
\hline Izquierda & 13,4 & 32,2 & 72,3 & 57,6 & 14,3 & 10,3 & $100(1.037)$ & $100(1.331)$ \\
\hline Centro & 8,1 & 26,0 & 80,6 & 61,3 & 11,3 & 12,7 & $100(397)$ & $100(354)$ \\
\hline Derecha & 9,6 & 14,1 & 62,7 & 35,9 & 27,7 & 50,0 & $100(271)$ & $100(64)$ \\
\hline Total & 11,6 & 30,2 & 72,7 & 57,5 & 15,7 & 12,2 & 100 & 100 \\
\hline (N columna) & (197) & (529) & $(1.240)$ & (1.006) & (268) & (214) & (1.705) & (1.749) \\
\hline
\end{tabular}

Postmaterial

$2006(3)$

\begin{tabular}{lrrrrrrrr} 
0. Materialismo & 19,2 & 11,9 & 71,2 & 25,4 & 9,6 & 62,7 & $100(292)$ & $100(59)$ \\
1. & 18,2 & 27,1 & 66,0 & 43,9 & 15,7 & 29,0 & $100(483)$ & $100(321)$ \\
2. & 10,5 & 24,2 & 77,0 & 62,8 & 12,5 & 13,0 & $100(861)$ & $100(938)$ \\
3. & 13,2 & 32,4 & 69,9 & 58,1 & 16,9 & 9,5 & $100(491)$ & $100(494)$ \\
4. & 10,0 & 51,9 & 61,1 & 39,4 & 28,9 & 8,7 & $100(90)$ & $100(264)$ \\
5. Postmaterial & 12,0 & 58,3 & 60,0 & 37,5 & 28,0 & 4,2 & $100(25)$ & $100(24)$ \\
\hline Total & 13,9 & 30,1 & 71,5 & 54,5 & 14,6 & 15,4 & 100 & 100 \\
(N columna) & $(311)$ & $(632)$ & $(1.603)(1.145)$ & $(328)$ & $(323)$ & $(2.242)$ & $(2.100)$
\end{tabular}

\begin{tabular}{lllll}
\hline (1) & Chi-cuadrado & GL & Sig. & Coef. Conting. \\
Andalucía & 59,975 & 4 & 0,000 & 0,190 \\
Cataluña & 33,406 & 4 & 0,000 & 0,145 \\
\hline (2) & Chi-cuadrado & GL & Sig. & Coef. Conting. \\
Andalucía & 46,182 & 4 & 0,000 & 0,162 \\
Cataluña & 94,099 & 4 & 0,000 & 0,226 \\
\hline (3) & Chi-cuadrado & GL & Sig. & Coef. Conting. \\
Andalucía & 53,943 & 10 & 0,000 & 0,153 \\
Cataluña & 256,836 & 10 & 0,000 & 0,330 \\
\hline
\end{tabular}

Fuente: ASEP y elaboración propia.

var algunos cambios interesantes. Los nacionalistas andaluces siguen declarándose de izquierdas, pauta que también siguen ahora los catalanes. Las personas con identidad dual se posicionan ahora en el centro en lugar de en la izquierda en ambas regiones. Por último, los españolistas andaluces se concentran en la derecha, y los catalanes se congregan en la derecha y, en menor medida, en el centro (pero no ya en la izquierda). 
Tabla 8. Sentimiento nacionalista según índice de posición social (totales en filas)

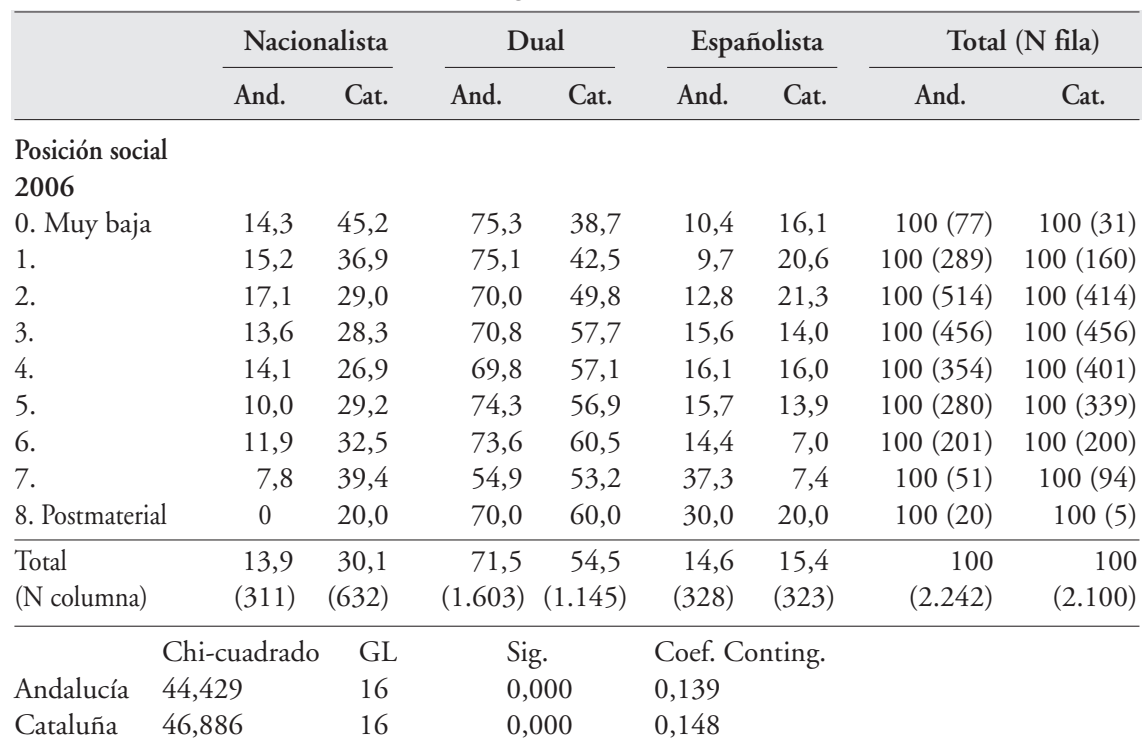

Fuente: ASEP y elaboración propia.

La tabla 8 recoge la distribución del sentimiento nacionalista teniendo en cuenta el índice de posición social elaborado por Galtung? ${ }^{9}$. Esta variable no es relevante en Cataluña en 1995, pero sí en 2006, mientras que en Andalucía disminuye su capacidad explicativa a lo largo de la década, aunque el modelo siga siendo significativo. En 1995, los nacionalistas andaluces se situaban en las posiciones muy bajas. Las personas con identidad dual se repartían entre diversas categorías, por lo que es difícil extraer conclusiones sobre ella. En cambio, las personas españolistas tendían a aumentar de importancia conforme se subía de categoría.

En 2006, se repite la pauta de que los nacionalistas andaluces ocupan las posiciones más bajas de la sociedad, lo que confirma algo comentado anteriormente al analizar el nivel educativo, que es una variable más sencilla que el índice de posición social: el nacionalismo andaluz históricamente se ha concentrado en sectores sociales con poco prestigio, con poca visibilidad, y por ello su consolidación en el espacio político no ha seguido pautas similares al catalán (para un análisis del sesgo popular del andalucismo y de la hegemonía social de los valores políticos, ver Del Pino y Bericat, 1998). Siguiendo con

9. Se ha cruzado el sentimiento nacionalista con el sexo, pero únicamente ha sido significativo en un solo caso: Andalucía en 1995, por lo que no se incluye aquí. En dicho año, las mujeres andaluzas son más nacionalistas y menos españolistas que los varones, mientras que la identidad dual se distribuye de manera uniforme entre hombres y mujeres. 
Andalucía, de nuevo encontramos a personas con mayor identidad dual en diversas categorías, mientras que el españolismo se vuelve a concentrar, de nuevo, en las posiciones más elevadas. En cuanto al caso catalán, existen algunos elementos en común y otros divergentes: los nacionalistas catalanes se concentran en las posiciones de ambos extremos, aunque no en la más alta. La identidad dual tiende a agruparse en las categorías intermedias, mientras que los españolistas se encuentran principalmente en las categorías más bajas. Si se compara el perfil de quienes se sienten sobre todo o únicamente españoles en Cataluña y en Andalucía, se observa que la visibilidad y el poder social de los primeros es mucho menor que el de los segundos.

Por último, la tabla 9 recoge la relación entre el sentimiento nacionalista y el hábitat y entre el primero y el estatus ocupacional. La relación entre sentimiento nacionalista y tamaño de hábitat difiere casi siempre en ambas comunidades: en Andalucía, en 1995, los nacionalistas se concentraban en el ámbito rural y semiurbano, mientras que en Cataluña únicamente en el primero; los andaluces con identificación dual se agrupaban en el ámbito rural, y en el semiurbano en el caso catalán; por último, los españolistas se concentran en el ámbito urbano andaluz y en el semiurbano catalán. Este último hecho probablemente se deba, si la hipótesis formulada anteriormente acerca de la relación entre españolismo e ideología es correcta, a que los emigrantes españoles se concentran en los cinturones industriales. En 2006, las pautas se repiten en el caso de los nacionalistas, pero existen cambios en la población con identidad dual: los andaluces con esta identificación se concentran ahora tanto en el ámbito rural como en el urbano, mientras que los catalanes habitan en municipios mayores de 100.000 habitantes. Por último, los españolistas catalanes siguen concentrándose en el ámbito semiurbano, una pauta que ahora se observa también en el caso andaluz.

El estatus ocupacional ha cobrado relevancia con el paso de la década para explicar el sentimiento nacionalista, especialmente en el caso de Cataluña. No obstante, la mayor parte de las pautas que se observan en 1995 se repiten una década después. En 1995, la población nacionalista andaluza se agrupaba en las categorías de menor nivel: estatus ocupacional bajo, ama de casa y en paro. En Cataluña, es más interesante destacar cuáles son los grupos que se sienten nacionalistas en menor medida, que son precisamente las personas en paro y las amas de casa. Las personas con identidad dual en Andalucía son, sobre todo, jubilados, estudiantes y amas de casa, por lo que se trata también de categorías bajas, aunque no coincidan exactamente con las anteriores. El perfil catalán coincide en parte, pues se concentra en amas de casa y personas en paro, precisamente las características de los nacionalistas andaluces. Por último, los españolistas en Andalucía pertenecen al estatus alto y medio y, en menor medida, son estudiantes y jubilados. En Cataluña, en cambio, las categorías donde se concentran son las de estatus bajo, parados, amas de casa y jubilados. Encontramos aquí, pues, diferencias fundamentales que inciden en lo ya comentado anteriormente sobre el perfil de los nacionalistas y los españolistas en ambas comunidades. 
Tabla 9. Sentimiento nacionalista según tamaño del hábitat y estatus ocupacional (totales en filas)

\begin{tabular}{|c|c|c|c|c|c|c|c|c|}
\hline & \multicolumn{2}{|c|}{ Nacionalista } & \multicolumn{2}{|c|}{ Dual } & \multicolumn{2}{|c|}{ Españolista } & \multicolumn{2}{|c|}{ Total (N fila) } \\
\hline & And. & Cat. & And. & Cat. & And. & Cat. & And. & Cat. \\
\hline \multicolumn{9}{|l|}{ Hábitat } \\
\hline Rural & 23,4 & 39,6 & 66,3 & 41,8 & 10,2 & 18,6 & $100(576)$ & $100(404)$ \\
\hline Semiurbano & 24,6 & 25,5 & 63,0 & 46,8 & 12,3 & 27,7 & 100 (901) & $100(654)$ \\
\hline Urbano & 21,3 & 29,0 & 63,4 & 46,4 & 15,3 & 24,7 & $100(888) 1$ & $100(1.005)$ \\
\hline $\begin{array}{l}\text { Total } \\
\text { (N columna) }\end{array}$ & $\begin{array}{c}23,1 \\
(546) \\
\end{array}$ & $\begin{array}{r}30,0 \\
(618) \\
\end{array}$ & $\begin{array}{r}64,0 \\
(1.513) \\
\end{array}$ & $\begin{array}{r}45,6 \\
(941) \\
\end{array}$ & $\begin{array}{r}12,9 \\
(306) \\
\end{array}$ & $\begin{array}{r}24,4 \\
(504) \\
\end{array}$ & $\begin{array}{r}100 \\
(2.365) \\
\end{array}$ & $\begin{array}{r}100 \\
(2.063) \\
\end{array}$ \\
\hline $2006(2)$ & & & & & & & & \\
\hline Rural & 14,6 & 47,5 & 71,9 & 38,7 & 13,5 & 13,8 & $100(569)$ & $100(305)$ \\
\hline Semiurbano & 17,2 & 28,8 & 64,8 & 52,9 & 18,1 & 18,3 & $100(781)$ & $100(809)$ \\
\hline Urbano & 10,5 & 25,8 & 77,1 & 60,8 & 12,3 & 13,5 & $100(892)$ & $100(986)$ \\
\hline $\begin{array}{l}\text { Total } \\
\text { (N columna) }\end{array}$ & $\begin{array}{c}13,9 \\
(311)\end{array}$ & $\begin{array}{r}30,1 \\
(632)\end{array}$ & $\begin{array}{r}71,5 \\
(1.603)\end{array}$ & $\begin{array}{r}54,5 \\
(1.145)\end{array}$ & $\begin{array}{r}14,6 \\
(328)\end{array}$ & $\begin{array}{c}15,4 \\
(323)\end{array}$ & $\begin{array}{c}100 \\
(2.242)\end{array}$ & $\begin{array}{r}100 \\
(2.100)\end{array}$ \\
\hline
\end{tabular}

\section{Estatus ocupacional}

1995 (3)

\begin{tabular}{lccrrrrrr} 
Alto & 12,8 & 46,3 & 61,7 & 36,3 & 25,5 & 17,5 & $100(47)$ & $100(80)$ \\
Medio & 20,7 & 32,2 & 61,4 & 45,9 & 17,9 & 21,9 & $100(503)$ & $100(590)$ \\
Bajo & 25,3 & 31,5 & 62,3 & 40,4 & 12,3 & 28,1 & $100(154)$ & $100(89)$ \\
En paro & 30,5 & 23,4 & 60,7 & 50,9 & 8,8 & 25,7 & $100(377)$ & $100(214)$ \\
Ama de casa & 25,3 & 23,2 & 65,1 & 48,2 & 9,6 & 28,5 & $100(659)$ & $100(508)$ \\
Jubilado & 18,7 & 30,0 & 67,7 & 43,6 & 13,6 & 26,4 & $100(433)$ & $100(424)$ \\
Estudiante & 17,7 & 42,8 & 66,7 & 42,8 & 15,6 & 14,5 & $100(192)$ & $100(159)$ \\
\hline Total & 23,1 & 29,9 & 64,0 & 45,7 & 12,9 & 24,4 & 100 & 100 \\
(N columna) & $(546)$ & $(618)$ & $(1.513)$ & $(943)$ & $(306)$ & $(503)$ & $(2.365)$ & $(2.064)$ \\
\hline 2006 (4) & & & & & & & & \\
Alto & 7,3 & 30,5 & 70,7 & 56,7 & 22,0 & 12,8 & $100(123)$ & $100(141)$ \\
Medio & 15,0 & 35,0 & 68,9 & 55,2 & 16,1 & 9,8 & $100(553)$ & $100(600)$ \\
Bajo & 14,8 & 20,4 & 74,0 & 61,1 & 11,2 & 18,5 & $100(384)$ & $100(368)$ \\
En paro & 21,2 & 21,9 & 70,5 & 63,8 & 8,3 & 14,3 & $100(193)$ & $100(105)$ \\
Ama de casa & 12,5 & 25,7 & 75,2 & 53,0 & 12,3 & 21,3 & $100(456)$ & $100(315)$ \\
Jubilado & 9,1 & 32,5 & 75,7 & 48,8 & 15,2 & 18,8 & $100(374)$ & $100(453)$ \\
Estudiante & 18,7 & 46,6 & 57,6 & 46,6 & 23,7 & 6,8 & $100(139)$ & $100(103)$ \\
Otros & 0 & 14,3 & 75,0 & 42,9 & 25,0 & 42,9 & $100(4)$ & $100(7)$ \\
\hline Total & 13,8 & 30,0 & 71,7 & 54,6 & 14,5 & 15,4 & 100 & 100 \\
(N columna) & $(307)$ & $(628)$ & $(1.597)$ & $(1.142)$ & $(322)$ & $(322)$ & $(2.226)$ & $(2.092)$ \\
\hline
\end{tabular}

\begin{tabular}{lllll}
\hline (1) & Chi-cuadrado & GL & Sig. & Coef. Conting. \\
Andalucía & 10,263 & 4 & 0,036 & 0,066 \\
Cataluña & 27,282 & 4 & 0,000 & 0,114 \\
\hline (2) & Chi-cuadrado & GL & Sig. & Coef. Conting. \\
Andalucía & 32,267 & 4 & 0,000 & 0,119 \\
Cataluña & 66,151 & 4 & 0,000 & 0,175 \\
\hline (3) & Chi-cuadrado & GL & Sig. & Coef. Conting. \\
Andalucía & 50,197 & 12 & 0,000 & 0,144 \\
Cataluña & 46,943 & 12 & 0,000 & 0,149 \\
\hline (4) & Chi-cuadrado & GL & Sig. & Coef. Conting. \\
Andalucía & 52,302 & 14 & 0,000 & 0,152 \\
Cataluña & 74,342 & 14 & 0,000 & 0,185 \\
\hline
\end{tabular}

Fuente: ASEP y elaboración propia. 
En 2006, las categorías de los nacionalistas andaluces se mantienen, pero se añaden otras dos: el estatus ocupacional medio y, sobre todo, los estudiantes. Ya se ha comentado en otro lugar (tabla 6) que el perfil educativo de estas personas ha cambiado en Andalucía a lo largo de la década, hecho que se corrobora con estos datos. La distribución del nacionalismo catalán repite, en general, las tendencias anteriores, salvo que ahora, además de las amas de casa y los parados, son las personas con estatus ocupacional bajo los que no escogen la opción nacionalista, con lo cual se acentúa aún más su carácter de opción elegida por personas que ocupan posiciones prestigiosas. La identidad dual en Andalucía predomina en los sectores menos favorecidos, pues se unen a los jubilados de 1995 las amas de casa y los de estatus ocupacional bajo, mientras pierden importancia los estudiantes. En Cataluña también cambia parcialmente el perfil de estas personas, que se concentra ahora en individuos con estatus bajo y desempleados. Por último, los españolistas andaluces tienen estatus ocupacionales altos y medios o bien son jubilados y estudiantes, mientras que en Cataluña vuelven a concentrarse en el estatus bajo, las amas de casa y los jubilados.

\section{Conclusiones}

Se quieren corroborar las hipótesis enunciadas en el primer epígrafe: 1) si las variables empleadas en los análisis explican menos en 2006 que en 1995 porque ambas sociedades se han modernizado y el proceso de individuación se ha profundizado; 2) si explican peor el caso catalán que el andaluz porque Cataluña se encuentra en un estadio más avanzado de la modernización. Para ello, se analizará la tabla 10, que da una visión de conjunto de los análisis vistos hasta ahora.

En cuanto a la primera variable estudiada, el «espacio geográfico con el que se siente identificado en primer lugar» — de la que sólo se dispone de datos para 2006- de las nueve variables que se han cruzado con el espacio geográfico, en Andalucía no son significativos los modelos que incluyen el sexo y el hábitat, mientras que en Cataluña todos los modelos son significativos. De los siete modelos relevantes en ambas, la chi-cuadrado es mayor en cuatro ocasiones en Cataluña. Por lo tanto, no queda confirmada la hipótesis de que en la sociedad catalana las categorías de origen y de pertenencia expliquen peor esta identificación con un territorio. La explicación para ello se podría encontrar en que la emigración a Cataluña tuvo un perfil definido en cuanto a su nivel educativo, cualificación profesional, edad, etc., y los emigrantes no se repartieron de forma uniforme por toda la comunidad, sino que se concentraron en ciertas aglomeraciones industriales, por lo que no es sorprendente que todas las variables sean relevantes en el caso catalán. Desgraciadamente, no disponemos de datos de 1995 para comprobar la hipótesis de que eran aún más relevantes en el pasado.

En cuanto a la segunda variable estudiada, el «sentimiento nacionalista», indudablemente también en ella incide el fenómeno de la emigración que se 
Tabla 10. Resumen de las relaciones encontradas entre el espacio geográfico y el sentimiento nacionalista y el resto de variables

\begin{tabular}{|c|c|c|c|}
\hline Variables & $\begin{array}{l}\text { Espacio } \\
\text { geográfico (2006) }\end{array}$ & $\begin{array}{l}\text { Sentimiento } \\
\text { nacionalista (1995) }\end{array}$ & $\begin{array}{l}\text { Sentimiento } \\
\text { nacionalista (2006) }\end{array}$ \\
\hline \multirow[t]{2}{*}{ Sexo } & And: No signif. & And: $17,681^{* * *}$ & And: No signif. \\
\hline & Cat: $18,816^{* *}$ & Cat: No signif. & Cat: No signif. \\
\hline \multirow[t]{2}{*}{ Edad } & And: No signif. & And: No signif. & And: $21,022^{* *}$ \\
\hline & Cat: $46,807^{* *}$ & Cat: $13,344^{*}$ & Cat: $29,054^{* * *}$ \\
\hline \multirow[t]{2}{*}{ Nivel educativo } & And: $170,385^{* * *}$ & And: $44,427^{* * *}$ & And: $58,297^{* * *}$ \\
\hline & Cat: $110,691^{* * *}$ & Cat: $117,263^{* * *}$ & Cat: $109,510^{* * *}$ \\
\hline \multirow{2}{*}{$\begin{array}{l}\text { Indice de posición } \\
\text { social de Galtung }\end{array}$} & And: $241,448^{* * *}$ & And: $60,460^{* * *}$ & And: $44,429^{* * *}$ \\
\hline & Cat: $102,004^{* * *}$ & Cat: No signif. & Cat: $46,886^{* * *}$ \\
\hline \multirow[t]{2}{*}{ Estatus ocupacional } & And: $137,550^{* * *}$ & And: $50,197^{* * *}$ & And: $52,302^{* * *}$ \\
\hline & Cat: $155,099^{* * *}$ & Cat: $46,943^{* * *}$ & Cat: $74,342^{* * *}$ \\
\hline \multirow[t]{2}{*}{ Ideología } & And: $135,722^{* * *}$ & And: $59,975^{* * *}$ & And: $46,182^{* * *}$ \\
\hline & Cat: $42,459^{* * *}$ & Cat: $33,406^{* * *}$ & Cat: $94,099^{* * *}$ \\
\hline \multirow[t]{2}{*}{ Postmaterialismo } & And: $126,113^{* * *}$ & And: No signif. & And: $53,943^{* * *}$ \\
\hline & Cat: $219,429^{* * *}$ & Cat: $21,805^{*}$ & Cat: $256,836^{* * *}$ \\
\hline \multirow[t]{2}{*}{ Hábitat } & And: No signif. & And: $10,263^{*}$ & And: $32,267^{* * *}$ \\
\hline & Cat: $88,844^{* * *}$ & Cat: $27,282^{* * *}$ & Cat: $66,151^{* * *}$ \\
\hline \multirow{2}{*}{$\begin{array}{l}\text { Sentimiento } \\
\text { nacionalista }\end{array}$} & And: $228,512^{* * *}$ & & \\
\hline & Cat: $739,998^{* * *}$ & - & - \\
\hline
\end{tabular}

Fuente: ASEP y elaboración propia.

acaba de mencionar. La primera hipótesis postula que las variables explican peor los datos de 2006 que de 1995. Sin embargo, el número de modelos relevantes es mayor en la segunda fecha, lo que contradice la hipótesis. Analizando la chi-cuadrado, se observa que algunos factores han ganado peso y otros lo han perdido. Los más numerosos son los primeros (especialmente en el caso catalán, en que sólo el nivel educativo ha disminuido levemente su importancia). Por tanto, tampoco en este caso parece confirmarse la hipótesis de que las categorías sociales cada vez explican menos las actitudes y los comportamientos en las sociedades modernas.

Por último, se trata de comparar ambas comunidades en un momento dado, para comprobar si en Andalucía las variables explicativas son más relevantes que en Cataluña: en 1995, de las ocho variables estudiadas, cuatro tienen una mayor chi-cuadrado en el caso andaluz y cuatro en el catalán. Pero lo que resulta sorprendente es que, en 2006, todas las variables son más relevantes en el caso catalán que en el andaluz. Es decir, no sólo en ambas comunidades la mayor parte de las variables cada vez explican más, sino que es en la sociedad que presenta mayor índice de desarrollo económico y social donde el poder explicativo ha aumentado en mayor proporción. Destaca especialmente el crecimiento de dos variables en Cataluña: el índice de postmaterialismo 
y la ideología. Ello es lógico: dado que se está estudiando un fenómeno de carácter político, son las dos variables que recogen las actitudes vitales de los individuos las que más relevancia cobran, frente a otros factores de carácter más socioeconómico. En cuanto a Andalucía, es también el postmaterialismo quien sobresale por su crecimiento. Sin embargo, la hipótesis de Inglehart esbozada al principio se comprueba en el caso andaluz, pero no en el catalán, lo que aconseja tener en cuenta otros factores antes de suponer que los postmaterialistas se identificarán con España (el territorio más amplio de los que se podían elegir).

Por supuesto, algunas de las variables estudiadas muestran una fuerte asociación entre ellas (el nivel educativo y la edad, o el índice de posición social con el estatus ocupacional y la educación, por ejemplo). Por ello, se han realizado regresiones logísticas (no mostradas aquí) en que se han incluido como variables independientes dicotómicas todas las utilizadas aquí y se ha analizado su influencia en cada una de las categorías del sentimiento nacionalista, tratadas como variables dependientes (identidad dual, españolismo y nacionalismo). No es éste el momento de extendernos en estos resultados, baste mencionar que el análisis vuelve a confirmar que las variables independientes son más relevantes para explicar el fenómeno en 2006 que en 1995 y, para ambos años, en Cataluña más que en Andalucía. En lo que se refiere al nacionalismo, se corrobora la divergencia histórica en el perfil social de los nacionalistas andaluces y catalanes y su capacidad de movilización. Sin duda, uno de los factores que explican la diferente trayectoria de ambos nacionalismos es el grado en que fue y es adoptado por parte de las élites políticas, económicas y culturales - la intelligentsia autóctona-, lo que confirma la importancia de tener en cuenta el componente humano para estudiar los procesos de modernización de cualquier tipo (Solé, 1976). No obstante, se observan algunos cambios interesantes: en el caso catalán, los jóvenes de 18 a 34 años son menos nacionalistas que las personas de otras edades, mientras que en Andalucía este sentimiento es más frecuente entre los 18 y los 44 años, en población semiurbana y ya no es característico de las personas sin estudios.

Ambos fenómenos se pueden relacionar con la historia política reciente de estas comunidades: por un lado, a partir del 2003, la experiencia del Gobierno catalán del tripartito (ERC, PSOE y ICV-EUiA), integrado por dos fuerzas políticas de implantación estatal y una nacionalista, unidas por su ideología progresista y que han llevado a cabo la reforma del Estatuto, indica que la tradicional división entre partidos estatales y nacionalistas ha quedado, al menos de momento, obsoleta, y que los partidos estatales asentados en territorio catalán asumen en mayor o menor medida el discurso nacionalista, especialmente los de carácter progresista, lo que podría repercutir en que la gente más joven, socializada — al menos en parte- en este contexto político, no se sienta tan nacionalista como los mayores de 34 años. Ello (junto al auge de ERC en los últimos años en detrimento de $\mathrm{CiU}$ ) explica otra pauta encontrada en estas regresiones (y que ya se había observado en el análisis de contingencia, ver la tabla 7): mientras que, en 1995, los que eran de centro eran más nacio- 
nalistas que los de izquierdas, en 2006 ocurre al contrario. Por otra parte, el PSOE andaluz asumió progresivamente, especialmente a partir de su coalición de gobierno con el Partido Andalucista en 1996 y 2000, y después en solitario, el discurso nacionalista. No hay que infravalorar el hecho de que, después de casi tres décadas de gobierno socialista, Andalucía sigue siendo una de las regiones menos desarrolladas de España pese a sus indudables progresos. El nacionalismo tiene un fuerte poder integrador y legitimador que es usado por los poderes del Estado (en este caso autonómico) para reunir a todos los ciudadanos en defensa de unos intereses comunes y diferentes a los del resto de España, aunque ello no implique aspiraciones independentistas o secesionistas, sino mayores cotas de autonomía y mayores recursos para financiar las nuevas competencias $^{10}$.

En la política española, afectada en este caso por una tendencia que también se ha dado en otros países del mundo, se ha reavivado la cuestión nacional en los últimos años. La globalización origina, como reacción, un resurgir de los particularismos, como ya notara Castells (1998) hace una década. En este contexto, los discursos nacionalistas de diverso carácter (más o menos moderado) se han extendido y han impactado en la sociedad española, incluso en comunidades donde la cuestión había suscitado un gran consenso durante décadas. Por ello, no es de extrañar que las categorías sociales cobren ahora nueva relevancia y que no se cumplan los supuestos de la teoría de la modernización clásica, que defiende que el comportamiento y las actitudes son más difíciles de predecir cuanto más desarrollada está una sociedad, porque ha llegado a un mayor proceso de individuación. El análisis del caso catalán y el andaluz aquí mostrado nos obliga a revisar esta hipótesis.

\section{Referencias bibliográficas}

ANDERSON, B. (1983). Imaginated communities. Reflections on the origin and spread of nationalism. Londres: Verso.

BeCK, U. (1992). Risk Society. Towards a New Modernity. Londres: SAGE Publications.

CASTELls, M. (1998). La era de la información. Economía, sociedad y cultura. Vol. 2. El poder de la identidad. Madrid: Alianza Editorial.

DeUTSCH, K.W. (1981). Las naciones en crisis. México: FCE.

GALTUNG, J. (1964). «Foreign policy opinion as a function of social position». Journal of Peace Research, 3 (4), 206-231.

GARCÍA FAROLDI, L. (2009). «Nación y territorio: análisis comparado del sentimiento nacionalista y la pertenencia a territorios en Andalucía y España». En: GobernAdo ARRIBAS, R. (coord. y ed.) (2009). La modernización regional en España. Pamplona: Civitas, 235-264.

GOBERNADO ARRIBAS, R. (coord.) (1996). Análisis comparado de las estructuras sociales de Andalucía y Cataluña. Málaga: Universidades de Málaga y Almería.

10. Para un análisis del sentimiento nacionalista, el españolismo y la identificación dual en la población española en general y las diferencias entre las comunidades autónomas, ver García Faroldi (2009). 
HAYES, C.J.H. (1966). El nacionalismo. Una religión. México: Uheta.

HobSBAWM, E.J. (1992). Naciones y nacionalismo desde 1780. Barcelona: Crítica.

IngleHART, R. (1977). The silent revolution: Changing Values and Political Styles among Western Publics. New Jersey: Princeton University Press.

- (1990). Cultural Shift in Advanced Industrial Society. New Jersey: Princeton Universtiy Press.

Kedourie, E. (1961). Nationalism. Londres: Hutchinson.

Pino Artacho, J. del; Bericat Alastuey, E. (1998). Valores sociales en la cultura andaluza. Madrid: CIS. Colección Monografías.

SMITH, A.D. (1986). The ethnics origins of nations. Cambridge: Cambridge University Press.

- (1991). National identities. Londres: Penguin.

Solé, C. (1976). Modernización. Un análisis sociológico. Barcelona: Península.

- (1997). Acerca de la modernización, la modernidad y el riesgo. REIS, 1997, 80, 111 131.

Treiman, D. J. (1970). «Industrialitation and Social Stratification». En: LAUMANN, E.O. (ed.). Social stratification: Research and theory for the 1970s. Nueva York: The Bobbs-Merril Company, Inc., 207-234. 